ESAIM: Proceedings, Vol. 4, 1998, 83-96

Contrôle et Équations Aux DÉrivées Partielles

http://www.emath.fr/proc/Vol.4/

\title{
SOME APPLICATIONS OF BV FUNCTIONS IN OPTIMAL CONTROL AND CALCULUS OF VARIATIONS ${ }^{1}$
}

\author{
E. CASAS \\ Departamento de Matemática \\ Aplicada y Ciencias de la Computación \\ E.T.S.I. Industriales y de Telecomunicación, Universidad de Cantabria \\ Av. Los Castros s/n, 39071 Santander, Spain \\ K. KUNISCH \\ Institut für Mathematik, \\ Universität Graz \\ Heinrichstrasse 36, A-8010 Graz, Austria \\ C. POLA \\ Departamento de Matemáticas, \\ Estadística y Computación \\ Facultad de Ciencias, Universidad de Cantabria \\ Av. Los Castros s/n, 39071 Santander,Spain
}

Key Words : Optimal Control, Bounded Variation Functions.

AMS Subject Qualification : 49J20, 49M29, 35J25.

\footnotetext{
${ }^{1}$ This research was partially supported by the European Union, under HCM Project number ERBCHRXCT940471. The second author was partially supported by the Fonds zur Förderung der wissenschaftlichen Forschung, Austria. The two other authors were also partially supported by Dirección General de Investigación Científica y Técnica (Spain)
}

Article published by EDP Sciences and available at http://www.edpsciences.org/proc or http://dx.doi.org/10.1051/proc:1998022 


\begin{abstract}
In this paper we discuss the use of bounded variation functions in the study of some optimal control problems as well as in the calculus of variations. The bounded variation functions are well adapted to the study of parameter identification problems, such as the coefficients of an elliptic or parabolic operator. These functions are also convenient for the image recovery problems. These problems are well formulated in the space $B V(\Omega)$ in the sense that they have a solution under reasonable assumptions. The numerical approximation of these problems is interesting because of the non separability of the space $B V(\Omega)$. A very surprising fact is the influence of the chosen norm, among all the possible equivalent norms in $B V(\Omega)$, on the convergence of the numerical approximations. Indeed the approximation by piecewise constant functions fails if the norm is not properly chosen.
\end{abstract}




\section{A problem of calculus of variations}

The image enhancement or image recovery problems, which have recently received a considerable amount of attention, are an example of problems of calculus of variations that can be studied in the space of the functions of bounded variation. To briefly describe this problem let $z$ denote the grey values of an image which extends in a two dimensional domain $\Omega$. The values of $z$ correspond to the values of the initial image $u$ on $\Omega$ corrupted by noise and blurring. This relationship is expressed as

$$
z=T u+\eta,
$$

where $\eta$ represents noise and $T$ is a linear operator that describes the blurring. To recover $u$ from $z$ a regularized least squares functional is minimized:

$$
(\mathcal{C V}) \quad \min \int_{\Omega}|T u-z|^{2} d x+\beta \int_{\Omega}|\nabla u|,
$$

where $\beta>0$ is the positive regularization parameter and $\int_{\Omega}|\nabla u|$ denotes the $B V$-seminorm. The notation $\int_{\Omega}|\nabla u|$ is formal here and will be made precise in Section 3 where we summarize properties of the space of $B V(\Omega)$ functions that are relevant to the theory of this research. For the moment it suffices to recall that functions in $B V(\Omega)$ are not necessarily continuous. The use of $B V$ seminorms was, to our knowledge, proposed originally in Osher and Rudin [12] with the goal to dampen excessive oscillations in $z$ without smoothing sharp edges and corners that are contained in the original image $u$. This would occur if the $B V$-seminorm was replaced by $\int_{\Omega}|\nabla u|^{2} d x$, for example. Let us point out that besides some technical difficulties related to the space of $B V$-functions, the main theoretical and numerical difficulty relates to the lack of differentiability of the cost functional. The image reconstruction problem with $B V$-regularization was further investigated in Dobson and Santosa [5], Ito and Kunisch [8], Li and Santosa [9] and Rudin et al. [14], for example. The contributions in [5], $[9]$ and [14] using variations of the formulation in $(\mathcal{C V})$ provide substantial evidence that $B V$-regularization can be a very effective numerical technique for image enhancement. In [8] augmented Lagrangian algorithms are analyzed for $(\mathcal{C V})$ under the assumption that an additional regularization term of the form $\gamma \int_{\Omega}|\nabla u|^{2} d x$, with $\gamma$ small with respect to $\beta$, is introduced and an active set strategy based on a duality technique is proposed. In Acar and Vogel [1] $B V$ regularization is studied and the problem of nondifferentiability is circumvented by replacing $\int_{\Omega}|\nabla u|$ by $\int_{\Omega} \sqrt{|\nabla u|^{2}+\epsilon}$, for $\epsilon>0$. Several theoretical aspects have been left open in the abovementioned papers some of which will be discussed here.

\section{An optimal control problem}

As a special example of optimal control problems we are going to discuss the following problem of identification of parameters. Let us consider the state 
equation

$$
\left\{\begin{aligned}
-\operatorname{div}(u(x) \nabla y(x))=f(x) & \text { in } \Omega \\
y(x)=0 & \text { on } \Gamma,
\end{aligned}\right.
$$

where $\Gamma$ is the boundary of $\Omega, f \in L^{2}(\Omega)$ and $u$ is the parameter to be identified. We consider the control problem

$$
\min _{u \in K \cap B V(\Omega)} \int_{\Omega}\left|y_{u}(x)-y_{d}(x)\right|^{2} d x+\beta \int_{\Omega}|\nabla u|
$$

where $y_{d} \in L^{2}(\Omega)$ and $\beta>0$ are given. With $y_{u}$ we denote the solution of (1) and

$$
K=\left\{u \in L^{\infty}(\Omega): u_{\min } \leq u(x) \leq u_{\max }\right\},
$$

where $0<u_{\min }<u_{\max }<+\infty$.

It is well known that in general problem $(\mathcal{O C})$ has no solution if $\beta=0$; see Murat [10]. The fact that $K$ is bounded in $L^{\infty}(\Omega)$ is not sufficient to assure the existence of a solution. Additional regularity of $u$, allowing a compactness argument is necessary to guarantee existence. Previously this was achieved by including the term $\beta \int_{\Omega}|\nabla u(x)|^{p} d x$ in the cost functional, $p>1$, in place of the $B V$-seminorm considered in the above formulation. Minimization in the Sobolev spaces $W^{1, p}(\Omega)$ was studied by Banks and Kunisch [2]. Alternatively the choice $\beta=0$ was used together with the constraint $|\nabla u(x)| \leq c$; see Casas [3]. In the latter case, the Sobolev space $W^{1, \infty}(\Omega)$ is taken for the parameter $u$. The use of Sobolev spaces can lead to overregularization of the function $u$. For instance if piecewise continuous functions have to be identified, the space $B V(\Omega)$ seems to be more appropriate than Sobolev spaces because one can obtain the necessary compactness to prove existence of a solution and the induced regularity for the parameter $u$ is not too restrictive, since it includes piecewise continuous functions. For a previous paper dealing with $B V$-functions to identify parameters, the reader is referred as to Gutman [7]. In that paper, theoretical questions remained open, which will be studied in this paper.

\section{The space $B V(\Omega)$}

Let $\Omega$ be an open bounded domain in $\mathbb{R}^{n}$ with a Lipschitz boundary $\Gamma$. With $M(\Omega)$ we denote the space of real Borel measures in $\Omega$, which is a Banach space when endowed with the norm

$$
\|\mu\|_{M(\Omega)}=|\mu|(\Omega)=\int_{\Omega}|\mu|
$$

$|\mu|=\mu^{+}+\mu^{-}$being the total variation measure associated to $\mu$ and then $|\mu|(\Omega)$ is the total variation of $\mu$ in $\Omega$. It is well known that $M(\Omega)$ is the dual of the space of the continuous functions in $\Omega$ vanishing on $\Gamma, C_{0}(\Omega)$, and

$$
\int_{\Omega}|\mu|=\sup \left\{\int_{\Omega} v(x) d \mu(x), v \in C_{0}(\Omega),\|v\|_{\infty} \leq 1\right\} .
$$


See, for instance, Rudin [13] for details.

Now let us denote by $B V(\Omega)$ the space of functions of bounded variation in $\Omega$. Let us remind that a function $u \in L^{1}(\Omega)$ is said to be a bounded variation function if $\partial_{x_{i}} u \in M(\Omega)$ for every $1 \leq i \leq n$, where the derivatives are understood in the distributional sense, or equivalently $u$ is a bounded variation function if for every $1 \leq i \leq n$ we have

$$
\sup \left\{\int_{\Omega} u \partial_{x_{i}} v d x, v \in C_{0}^{\infty}(\Omega),\|v\|_{\infty} \leq 1\right\}=\int_{\Omega}\left|\partial_{x_{i}} u\right|<+\infty .
$$

Setting

$$
\|u\|_{B V(\Omega)}=\int_{\Omega}|u| d x+\sum_{i=1}^{n} \int_{\Omega}\left|\partial_{x_{i}} u\right|=\int_{\Omega}|u| d x+\int_{\Omega}|\nabla u|,
$$

we have that $B V(\Omega)$ is a Banach space. Let us note that (5) can be written as

$$
\begin{gathered}
\|u\|_{B V(\Omega)}=\int_{\Omega}|u| d x \\
+\sup \left\{\int_{\Omega} u(x) \operatorname{div} v(x) d x, v \in C_{0}^{\infty}(\Omega)^{n},|v(x)|_{\infty} \leq 1, x \in \Omega\right\}
\end{gathered}
$$

where $|\cdot|_{\infty}$ denotes the $l_{\infty}$ norm in $\mathbb{R}^{n}$ :

$$
|x|_{\infty}=\max _{1 \leq i \leq n}\left|x_{i}\right| .
$$

A different norm in $\mathbb{R}^{n}$ induces another norm on $B V(\Omega)$ that is equivalent to (5). For instance, a frequently used norm, mainly employed in the study of minimal surfaces, is the Euclidean norm, see Giusti [6]. The reader is referred to Temam [15] for the use of the norm (5).

The proof of the next properties of $B V(\Omega)$ can be found in [6].

Proposition 3.1 1) If $\left\{u_{j}\right\}_{j=1}^{\infty} \subset B V(\Omega)$ and $u_{j} \rightarrow u$ in $L^{1}(\Omega)$, then

$$
\int_{\Omega}\left|\partial_{x_{i}} u\right| \leq \liminf _{j \rightarrow \infty} \int_{\Omega}\left|\partial_{x_{i}} u_{j}\right| .
$$

2) For every $u \in B V(\Omega) \cap L^{r}(\Omega), r \in[1,+\infty)$, there exists a sequence $\left\{u_{j}\right\}_{j=1}^{\infty} \subset C^{\infty}(\bar{\Omega})$ such that

$$
\lim _{j \rightarrow \infty} \int_{\Omega}\left|u-u_{j}\right|^{r} d x=0 \text { and } \lim _{j \rightarrow \infty} \int_{\Omega}\left|\partial_{x_{i}} u_{j}\right|=\int_{\Omega}\left|\partial_{x_{i}} u\right|, \quad 1 \leq i \leq n .
$$

3) For every bounded sequence $\left\{u_{j}\right\}_{j=1}^{\infty} \subset B V(\Omega)$ there exists a subsequence $\left\{u_{j_{k}}\right\}_{k=1}^{\infty}$ and a function $u \in B V(\Omega)$ such that $u_{j_{k}} \rightarrow u$ in $L^{1}(\Omega)$.

ESAIM: Proc., Vol. 4, 1998, 83-96 
Concerning 2) one can generalize Giusti [6, Theorem 1.17] from $r=1$ to $r \in[1,+\infty)$ to assert that $C^{\infty}(\Omega) \cap L^{r}(\Omega)$ approximates $B V(\Omega) \cap L^{r}(\Omega)$ in the sense of (7). This implies that $W^{1,1}(\Omega) \cap L^{r}(\Omega)$ approximates $B V(\Omega) \cap L^{r}(\Omega)$ in the sense of $(7)$ as well. Since $\Gamma$ is Lipschitz, $C^{\infty}(\bar{\Omega})$ is dense in $W^{1,1}(\Omega) \cap$ $L^{r}(\Omega)$ (see, for instance, Nečas [11]), and we conclude that $C^{\infty}(\bar{\Omega})$ approximates $B V(\Omega) \cap L^{r}(\Omega)$ as claimed.

Let us remark that $(7)$ can not be modified to have $\left\|\partial_{x_{i}} u_{j}-\partial_{x_{i}} u\right\|_{M(\Omega)} \rightarrow 0$. Indeed the closure of $C^{\infty}(\bar{\Omega})$ in the norm $(5)$ is the Sobolev space $W^{1,1}(\Omega)$, which is obviously a strict subspace of $B V(\Omega)$, with

$$
\|u\|_{W^{1,1}(\Omega)}=\|u\|_{B V(\Omega)} \text { for all } u \in W^{1,1}(\Omega) .
$$

\section{Existence of solution}

In this section we study the existence of a solution for problem

$$
\min _{u \in K} F(u)=J(u)+\beta \int_{\Omega}|\nabla u|,
$$

where $\beta>0, K$ is a convex closed subset of $L^{p}(\Omega)(1 \leq p<+\infty)$, and $J: K \longrightarrow$ $\mathbb{R}$ is a weakly lower semicontinuous function. We also assume that $K \cap B V(\Omega)$ is nonempty and that $J$ is bounded from below.

The following theorem provides the necessary conditions to guarantee the existence of a solution for $(\mathrm{P})$.

Theorem 4.1 Let us suppose that either $K$ is bounded in $L^{p}(\Omega)$ or $J$ is coercive in the following sense: If $\left\{u_{j}\right\}_{j=1}^{\infty} \subset K,\left\{\int_{\Omega}\left|\nabla u_{j}\right|\right\}_{j=1}^{\infty}$ is bounded and $\left\|u_{j}\right\|_{L^{p}(\Omega)} \rightarrow+\infty$, then $J\left(u_{j}\right) \rightarrow+\infty$. Then $(\mathrm{P})$ has at least one solution $\bar{u}$. Moreover, if $J$ is a strictly convex function, then the solution is unique.

Proof: Let $\left\{u_{j}\right\}_{j=1}^{\infty} \subset K$ be a minimizing sequence, i.e. $F\left(u_{j}\right) \searrow \inf (\mathrm{P})$. Since $J$ is bounded from below it follows that

$$
\beta \int_{\Omega}\left|\nabla u_{j}\right|=F\left(u_{j}\right)-J\left(u_{j}\right) \leq F\left(u_{1}\right)-C<+\infty, \text { for all } j
$$

and some appropriately chosen constant $C$, and hence $\left\{\int_{\Omega}\left|\nabla u_{j}\right|\right\}_{j=1}^{\infty}$ is bounded. If $K$ is bounded or if $J$ satisfies the coercivity assumption of the above statement, we have that $\left\{u_{j}\right\}_{j=1}^{\infty}$ is bounded in $L^{p}(\Omega)$ as well.

We have proved that $\left\{u_{j}\right\}_{j=1}^{\infty}$ is bounded in $B V(\Omega) \cap L^{p}(\Omega)$. Therefore by using Proposition 3.1, we can take a subsequence that we will denote in the same way, and an element $\bar{u} \in B V(\Omega) \cap L^{p}(\Omega)$ such that

$$
\lim _{j \rightarrow \infty} u_{j}=\bar{u} \text { weakly in } L^{p}(\Omega) \quad \text { and } \quad \int_{\Omega}|\nabla \bar{u}| \leq \liminf _{j \rightarrow \infty} \int_{\Omega}\left|\nabla u_{j}\right| .
$$

Now using the weak lower semicontinuity of $J$, we get

$$
F(\bar{u}) \leq \liminf _{j \rightarrow \infty} F\left(u_{j}\right)=\inf (\mathrm{P}) .
$$


Since $K$ is closed in the weak topology of $L^{p}(\Omega), \bar{u} \in K$, which along with the previous inequality proves that $\bar{u}$ is a solution of $(\mathrm{P})$.

The uniqueness of the solution under the strict convexity of $J$ follows by using the classical argument.

Let us remark that the continuity of $J$ is enough to conclude the existence of a solution if $K$ is a bounded subset of $L^{\infty}(\Omega)$. Indeed, the boundedness of $\left\{u_{j}\right\}_{j=1}^{\infty}$ in $B V(\Omega)$ implies (Proposition 3.1) the existence of a subsequence, denoted in the same way, and an element $\bar{u} \in B V(\Omega)$ such that $u_{j} \rightarrow \bar{u}$ strongly in $L^{1}(\Omega)$. This together with the boundedness of $\left\{u_{j}\right\}_{j=1}^{\infty}$ in $L^{\infty}(\Omega)$ implies strong convergence $u_{j} \rightarrow \bar{u}$ in $L^{p}(\Omega)$, so that $J\left(u_{j}\right) \rightarrow J(\bar{u})$.

It is an easy exercise to prove that the above theorem can be applied to the study of problems $(\mathcal{C V})$ and $(\mathcal{O C})$ to deduce the existence of a solution.

\section{Approximation of Problem (P)}

Two issues are going to be addressed in this section. The first is concerned with the approximation of the space $B V(\Omega)$ by finite dimensional spaces. Secondly we will consider the approximation of problem $(\mathrm{P})$. To deal with the approximation of $B V(\Omega)$ we introduce a decomposition of $\Omega$ in subdomains. Since $\Omega$ is bounded we can take for every $1 \leq k \leq n$

$$
a_{k}=\min \left\{x_{k}: x=\left(x_{j}\right)_{j=1}^{n} \in \bar{\Omega}\right\} b_{k}=\max \left\{x_{k}: x=\left(x_{j}\right)_{j=1}^{n} \in \bar{\Omega}\right\} .
$$

Now we define

$$
D=\prod_{k=1}^{n}\left(a_{k}, b_{k}\right) \supset \Omega .
$$

Every interval $\left[a_{k}, b_{k}\right]$ is divided in $m_{k}$ subintervals:

$$
a_{k}=t_{k 0}<t_{k 1}<\cdots<t_{k m_{k}}=b_{k},
$$

and we take

$$
D_{k_{1} \cdots k_{n}}=\left(t_{1 k_{1}-1}, t_{1 k_{1}}\right) \times \cdots \times\left(t_{n k_{n}-1}, t_{n k_{n}}\right) .
$$

In order to simplify the notations, we denote $\tilde{m}=m_{1} \times \cdots \times m_{n}$ and rename the $\tilde{m}$ parallelepipeds $D_{k_{1} \cdots k_{n}}$ as $D_{j}, 1 \leq j \leq \tilde{m}$. Furthermore we put

$$
D_{j}=\prod_{k=1}^{n}\left(a_{k j}, b_{k j}\right)
$$

where every interval $\left(a_{k j}, b_{k j}\right)$ corresponds to some interval $\left(t_{k i_{k}-1}, t_{k i_{k}}\right)$. Thus we have

$$
\bar{D}=\bigcup_{j=1}^{\tilde{m}} \bar{D}_{j} \quad \text { and } \quad D_{i} \bigcap D_{j}=\emptyset \text { if } i \neq j .
$$

Let us notice that some of the parallelepipeds $D_{j}$ can have empty intersection with $\Omega$. Let us enumerate the family of parallelepipeds $\left\{D_{j}\right\}_{j=1}^{\tilde{m}}$ in such a way 
that $D_{j} \cap \Omega \neq \emptyset$ for $1 \leq j \leq m$ and $D_{j} \cap \Omega=\emptyset$ if $j>m$, with $m \leq \tilde{m}$. Now we take $\Omega_{j}=D_{j} \cap \Omega$ for $j=1, \cdots, m$. As a consequence of (12) we have

$$
\bar{\Omega}=\bigcup_{j=1}^{\tilde{m}} \bar{\Omega}_{j} \quad \text { and } \quad \Omega_{i} \bigcap \Omega_{j}=\emptyset \text { if } i \neq j .
$$

In the one dimensional case the situation is much simpler:

$$
\bar{\Omega}=[a, b]=\bigcup_{j=1}^{m}\left[a_{j}, b_{j}\right]=\bigcup_{j=1}^{m} \bar{\Omega}_{j}, a=a_{1}, \quad b=b_{m}, \quad a_{j}=b_{j-1}, \quad 2 \leq j \leq m .
$$

We associate with these domains $\left\{\Omega_{j}\right\}_{j=1}^{m}$ a finite dimensional space

$$
V_{m}=\left\{u_{m}=\sum_{j=1}^{m} u^{j} \chi_{\Omega_{j}}: u^{j} \in \mathbb{R}, 1 \leq j \leq m\right\},
$$

where $\chi_{\Omega_{j}}$ denotes the characteristic function of $\Omega_{j} . V_{m}$ is the space of piecewise constant functions associated with the partition $\left\{\Omega_{j}\right\}_{j=1}^{m}$ of $\Omega$. The following theorem states that any element of $V_{m}$ is a function of bounded variation and characterizes its total variation. Before stating the theorem we need to introduce some additional notation.

For $n>1$ and each $j$ we denote by $\partial \Omega_{j}$ the boundary of $\Omega_{j}$ and $\nu_{j}(x)$ stands for the outward normal vector (in the sense of the Euclidean scalar product) to $\partial \Omega_{j}$ at the point $x \in \partial \Omega_{j}$, which exists for almost every point of the boundary. We set $\left|\Omega_{j}\right|$ (resp. $\left.\left|\partial \Omega_{j}\right|\right)$ to indicate the $n$-dimensional (resp. $(n-1)$-dimensional) Lebesgue measure of $\Omega_{j}$ and with $S_{j}$ we represent the $(n-1)$-measure on the manifold $\partial \Omega_{j} \cap \Omega$, so that $\left|\partial \Omega_{j} \cap \Omega\right|=S_{j}\left(\partial \Omega_{j} \cap \Omega\right)$.

Now given two domains $\Omega_{i}$ and $\Omega_{j}$ such that $\left|\partial \Omega_{j} \cap \partial \Omega_{i}\right|>0$, we denote by $S_{i j}$ the $(n-1)$-measure on the manifold $\partial \Omega_{j} \cap \partial \Omega_{j}$ and by $\nu_{i j}$ the unit normal vector to $\partial \Omega_{i} \cap \partial \Omega_{j}$ pointing from $\Omega_{i}$ into $\Omega_{j}$. It is obvious that $\nu_{i j}(x)=\nu_{i}(x)$ for every $x \in \partial \Omega_{i} \cap \partial \Omega_{j}$ and that $\nu_{i j}=-\nu_{j i}$. The following theorem provides the total variation of the functions of $V_{m}$. 
Theorem 5.1 For every $m \in \mathbb{N}$ the inclusion $V_{m} \subset B V(\Omega)$ holds and for each $u_{m} \in V_{m}$

$$
\int_{\Omega}\left|\nabla u_{m}\right|= \begin{cases}\sum_{j=2}^{m}\left|u^{j}-u^{j-1}\right| & \text { if } n=1, \\ \sum_{i<j}\left|u^{i}-u^{j}\right|\left|\partial \Omega_{i} \cap \partial \Omega_{j}\right| & \text { if } n>1 .\end{cases}
$$

The next theorem establishes that $B V(\Omega)$ can be conveniently approximated by the spaces $V_{m}$. Let us define

$$
h_{m}=\max \left\{b_{k j}-a_{k j}: 1 \leq j \leq m, \quad 1 \leq k \leq n\right\} .
$$

Theorem 5.2 Let us assume that $h_{m} \rightarrow 0$ when $m \rightarrow \infty$. Then for every $u \in B V(\Omega) \cap L^{r}(\Omega), 1 \leq r<+\infty$, we can find a sequence $\left\{u_{m}\right\}$, with $u_{m} \in V_{m}$, such that

$$
\lim _{m \rightarrow \infty} \int_{\Omega}\left|u-u_{m}\right|^{r} d x=0 \quad \text { and } \lim _{m \rightarrow \infty} \int_{\Omega}\left|\nabla u_{m}\right|=\int_{\Omega}|\nabla u| .
$$

Remark 5.3 As we mentioned in $\S 1$, sometimes the following norm is considered in $B V(\Omega)$

$$
\|u\|=\int_{\Omega}|u(x)| d x+\int_{\Omega}|\nabla u|_{2}
$$

where

$$
\int_{\Omega}|\nabla u|_{2}=\sup \left\{\int_{\Omega} u(x) \operatorname{div} v(x) d x, v \in C_{0}^{\infty}(\Omega)^{n},|v(x)|_{2} \leq 1, x \in \Omega\right\},
$$

with $|\cdot|_{2}$ denoting the Euclidean norm in $\mathbb{R}^{n}$. Though (5) and (18) are equivalent norms, the approximation property (17) does not hold in general for the seminorm (19). Let us prove this fact. First we remark that

$$
\int_{\Omega}|\nabla u|_{2} \leq \sum_{k=1}^{n} \int_{\Omega}\left|\partial_{x_{k}} u\right| \text { for all } u \in B V(\Omega),
$$

the inequality being strict in general. This can be easily checked for the functions of $W^{1,1}(\Omega)$ because

$$
|x|_{2}<\sum_{k=1}^{n}\left|x^{k}\right| \quad \text { for every } x \in \mathbb{R}^{n}
$$

assuming that $x$ is not collinear with any vector $e_{k}$ of the canonical base. Every function of $B V(\Omega)$ can be approximated by functions of $W^{1,1}(\Omega)$ in the sense of (7). The property

$$
\int_{\Omega}|\nabla u|_{2} \leq \liminf _{m \rightarrow \infty} \int_{\Omega}\left|\nabla u_{m}\right| d x
$$

ESAIM: Proc., Vol. 4, 1998, 83-96 
holds whenever $\left\{u_{m}\right\} \subset B V(\Omega)$ and converges to $u$ in $L^{1}(\Omega)$, see Giusti [6]. This leads to $(20)$, with the inequality being strict in general.

Now we show that the approximation property (17) does not hold, in general, for the seminorm of (19). Let us take $u \in B V(\Omega)$ such that (20) holds strictly and arguing by contradiction let us assume that there exist a sequence $\left\{u_{m}\right\}$, with $u_{m} \in V_{m}$, such that

$$
\lim _{m \rightarrow \infty} \int_{\Omega}\left|u(x)-u_{m}(x)\right| d x=0 \text { and } \lim _{m \rightarrow \infty} \int_{\Omega}\left|\nabla u_{m}\right|_{2}=\int_{\Omega}|\nabla u|_{2} .
$$

We compute the total variation of $u_{m}$ in the sense of (19). To do this we take $v \in C_{0}^{\infty}(\Omega)^{n}$, with $|v(x)|_{2} \leq 1$ for every $x \in \Omega$. Then we have

$$
\begin{gathered}
\int_{\Omega} u_{m}(x) \operatorname{div} v(x) d x=\sum_{j=1}^{m} u^{j} \int_{\Omega_{j}} \operatorname{div} v(x) d x= \\
\sum_{j=1}^{m} u^{j} \int_{\partial \Omega_{j}} v(x) \nu_{j}(x) d S_{j}(x)=\sum_{i<j}\left(u^{i}-u^{j}\right) \int_{\partial \Omega_{j} \cap \partial \Omega_{i}} v(x) \nu_{i j}(x) d S_{i j}(x) .
\end{gathered}
$$

Taking the supremum in $v$, we get from the previous identity and (15) that

$$
\int_{\Omega}\left|\nabla u_{m}\right|_{2}=\sum_{i<j}\left|u_{i}-u_{j}\right|\left|\partial \Omega_{i} \cap \partial \Omega_{j}\right|=\sum_{k=1}^{n} \int_{\Omega}\left|\partial_{x_{k}} u_{m}\right| .
$$

By (6), we deduce form (21) and (22) that

$$
\sum_{k=1}^{n} \int_{\Omega}\left|\partial_{x_{k}} u\right| \leq \lim _{m \rightarrow \infty} \int_{\Omega}\left|\nabla u_{m}\right|_{2}=\int_{\Omega}|\nabla u|_{2}<\sum_{k=1}^{n} \int_{\Omega}\left|\partial_{x_{k}} u\right|,
$$

which gives the desired contradiction.

The key point in the above proof is (22), which holds because the vectors $\nu_{i j}$ coincide with the vectors of the canonical base of $\mathbb{R}^{n}$. Consequently $\left|\nu_{i j}\right|_{p}=1$ for every $1 \leq p \leq+\infty$, where $|\cdot|_{p}$ denotes the $l_{p}$ norm in $\mathbb{R}^{n}$. In fact we have that

$$
\begin{gathered}
\int_{\Omega}|\nabla u|_{p^{\prime}}=\sup \left\{\int_{\Omega} u(x) \operatorname{div} v(x) d x, v \in C_{0}^{\infty}(\Omega)^{n},|v(x)|_{p} \leq 1, x \in \Omega\right\} \leq \\
\int_{\Omega}|\nabla u|_{1}=\sum_{k=1}^{n} \int_{\Omega}\left|\partial_{x_{k}} u\right|,
\end{gathered}
$$

the inequality being strict in general. Here $p^{\prime}$ denotes the conjugate of $p$. Moreover

$$
\int_{\Omega}\left|\nabla u_{m}\right|_{p}=\sum_{i<j}\left|u_{i}-u_{j}\right|\left|\partial \Omega_{i} \cap \partial \Omega_{j}\right|, \quad \text { for every } 1 \leq p \leq \infty .
$$

Summarizing the discussion we found that among all the equivalent norms in $B V(\Omega)$ induced by the $l_{p}$ norms of $\mathbb{R}^{n}$, only the norm (5) considered in this paper has the approximation property (17) in the spaces $V_{m}$. 
We refer to[4] for additional results concerning the approximation of $B V(\Omega)$ by finite element subspaces.

Now we consider the approximation of problem $(\mathrm{P})$. In the remaining part of this section $V_{m}$ will denote a finite dimensional subspace of $B V(\Omega) \cap L^{p}(\Omega)$. For every $m$ we take $K_{m}=K \cap V_{m}$ and we make the following assumption

Assumption (A) For every $u \in K$ there exists a sequence $\left\{u_{m}\right\}$, with $u_{m} \in K_{m}$, such that

$$
\lim _{m \rightarrow \infty} \int_{\Omega}\left|u(x)-u_{m}(x)\right|^{p} d x=0 \text { and } \lim _{m \rightarrow \infty} \int_{\Omega}\left|\nabla u_{m}\right|=\int_{\Omega}|\nabla u| .
$$

If

$$
K=\left\{u \in L^{p}(\Omega): u_{\min } \leq u(x) \leq u_{\max }, \text { a.e. } x \in \Omega\right\},
$$

where $u_{\min }<u_{\max }$ are constants, then $K_{m}=K \cap V_{m}$ satisfies $(A)$ when $\left\{V_{m}\right\}$ is given as in Theorem 5.2.

Now we formulate the finite dimensional optimization problems

$$
\left(\mathrm{P}_{m}\right) \quad \min _{u_{m} \in K_{m}} F\left(u_{m}\right)=J\left(u_{m}\right)+\beta \int_{\Omega}\left|\nabla u_{m}\right| .
$$

The next theorem deals with the existence of a solution for these problems and with their convergence.

Theorem 5.4 Let us assume that $(A)$ holds and that either $K$ is bounded in $L^{p}(\Omega)$ or $J$ is coercive in the sense of Theorem 4.1. Then $\left(\mathrm{P}_{m}\right)$ has at least one solution $\bar{u}_{m}$ for every $m$. The sequence $\left\{\bar{u}_{m}\right\}$ is bounded in $L^{p}(\Omega) \cap B V(\Omega)$ and the limit of every subsequence converging weakly in $L^{p}(\Omega)$ also converges strongly in $L^{1}(\Omega)$ and it is a solution of $(\mathrm{P})$. If $J$ is strictly convex, then the solution of $\left(\mathrm{P}_{m}\right)$ is unique and the whole sequence $\left\{\bar{u}_{m}\right\}$ converges to the solution of $(\mathrm{P})$.

For the proof we refer to [4].

\section{Application to image enhancement}

We give a brief description and some numerical results of $(B V)$-seminorm regularization to the image denoising problem. Thus we consider the discretized form of $(\mathcal{P})$ of Section $\S 1$ with $T=I$. The unperturbed image $u^{*}$ extends over a square consisting of $80 \times 80$ pixels and the noisy image $z$ is obtained from

$$
z_{i j}=u_{i j}^{*}+\eta_{i j}, \quad i, j=1, \ldots, 80,
$$

with $\eta_{i j}$ uniformly distributed random numbers in $[-\delta, \delta]$. The use of the $(B V)$ seminorm regularization is expected to be especially effective for blocky images and thus as test example we considered, among others, $u^{*}=u_{1}^{*}+u_{2}^{*}+u_{3}^{*}$, where $u_{1, i, j}^{*}=1$ for all $i, j$,

$$
u_{2, i, j}^{*}= \begin{cases}1 & \text { if } 13 \leq i \leq 68 \text { and } 13 \leq j \leq 68 \\ 0 & \text { otherwise }\end{cases}
$$

ESAIM: Proc., Vol. 4, 1998, 83-96 


$$
u_{3, i, j}^{*}= \begin{cases}1 & \text { if } 25 \leq i \leq 56 \text { and } i \leq j \leq 56, \\ 0 & \text { otherwise }\end{cases}
$$

After reshaping the matrices $z$ and $u^{*}$ as $m \times 1$ vectors, $m=6400$, the resulting minimization problem can be expressed as

$$
\min _{u_{m} \in \mathbb{R}^{m}} \frac{1}{2 m} \sum_{i=1}^{m}\left|u_{i}-z_{i}\right|^{2}+\beta \sum_{i<j}\left|\partial \Omega_{i} \cap \partial \Omega_{j}\right|\left|u^{i}-u^{j}\right| .
$$

We considered a MATLAB code to solve (26). In Figure 1 we show the noisy image with $\delta=1$ and Figure 2 give the corresponding enhanced image obtained by the code. 
FIG. 1. The noisy image.

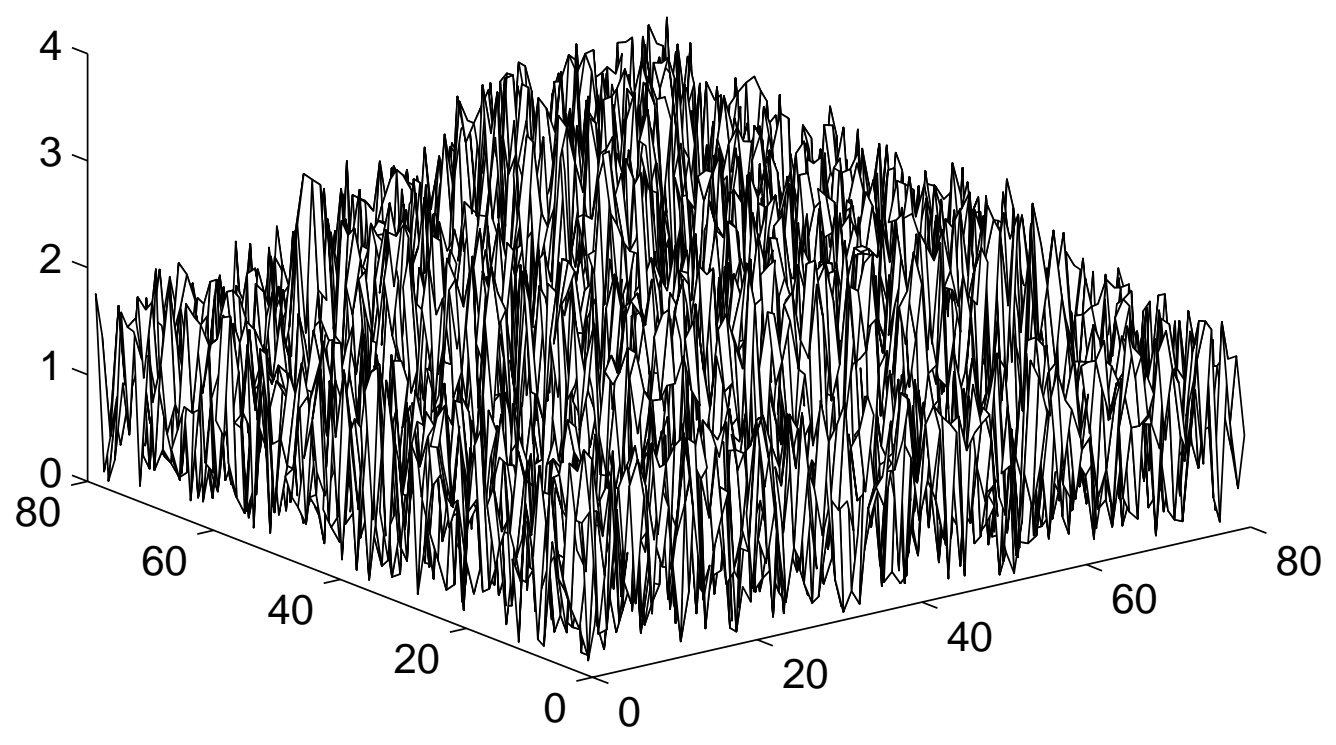

FIG. 2. Enhanced image.

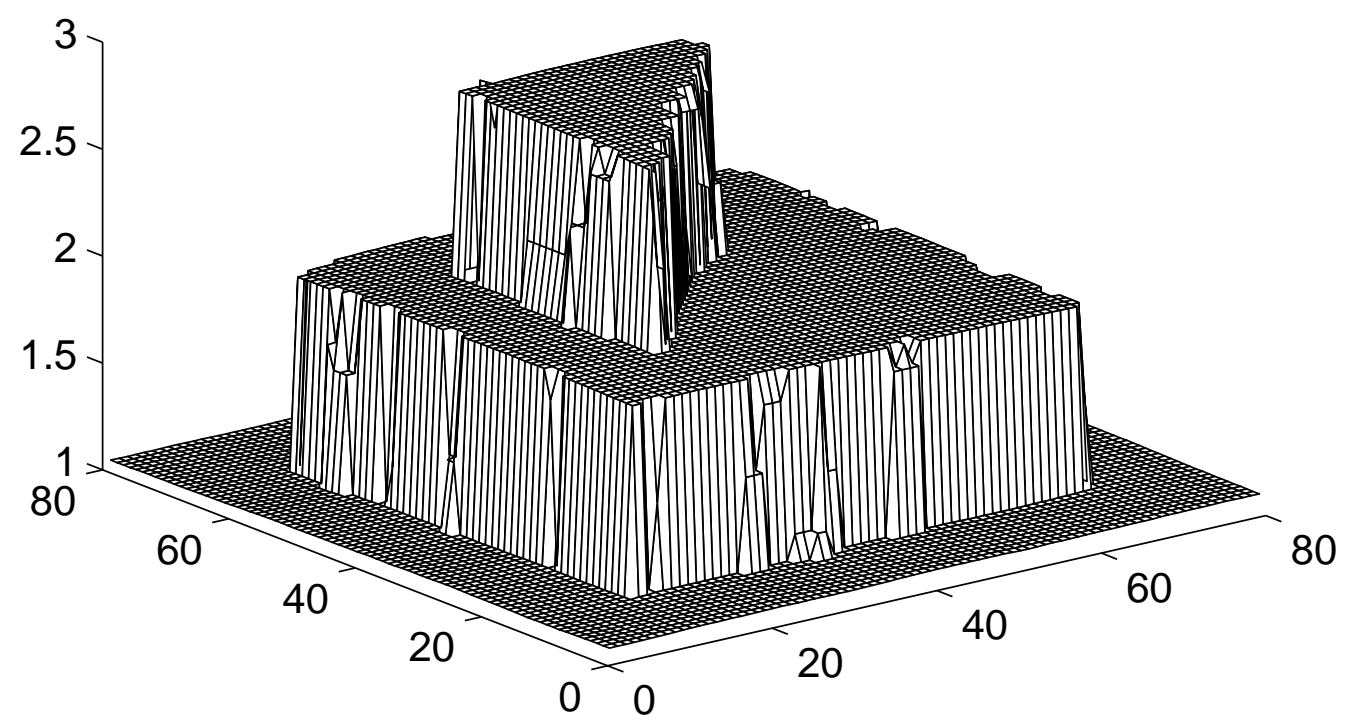

ESAIM: Proc., Vol. 4, 1998, 83-96 


\section{References}

[1] R. Acar and C.R. Vogel. Analysis of bounded variation penalty methods for ill-posed problems. Inverse Problems, 10:1217-1229, 1994.

[2] H.T. Banks and K. Kunisch. Estimation Techniques for Distributed Parameter Systems. Birkhäuser, Boston, 1989.

[3] E. Casas. Optimal control in coefficients with state constraints. Appl. Math. Optim., 26:21-37, 1992.

[4] E. Casas, K. Kunisch and C. Pola. Regularization by functions of bounded variation and applications to image enhancement. To appear in Appl. Math. Optim.

[5] D. Dobson and F. Santosa. Recovery of blocky images from noisy and blurred data. Technical Report 94-7, Department of Mathematical Sciences, University of Delaware, 1994.

[6] E. Giusti. Minimal Surfaces and Functions of Bounded Variation. Birkhäuser, Boston, 1984.

[7] S. Gutman. Identification of discontinuous parameters in flow equations. SIAM J. Control Optim., 28(5):1049-1060, 1990.

[8] K. Ito and K. Kunisch. An active set strategy based on the augmented Lagrangian formulation for image restoration. To appear in R.A.I.R.O. $M M A N$.

[9] Y. Li and F. Santosa. An affine scaling algorithm for minimizing total variation in image enhancement. Technical Report CTC94TR201, Cornell Theory Center, Cornell University, 1994.

[10] F. Murat. Contre exemple pour divers problèmes où le contrôle intervient dans les coefficients. Ann. Mat. Pur. Appl., 112:49-68, 1977.

[11] J. Nečas. Les Méthodes Directes en Théorie des Equations Elliptiques. Editeurs Academia, Prague, 1967.

[12] S. Osher and L. Rudin. Feature-oriented image enhancement using shock filters. SIAM J. Numer. Anal., 27:919-940, 1990.

[13] W. Rudin. Real and Complex Analysis. McGraw-Hill, London, 1970.

[14] L. Rudin, S. Osher, and C. Fu. Nonlinear total variation based noise removal algorithms. Physica D., 60:259-268, 1992.

[15] R. Temam. Mathematical Problems in Plasticity. Gauthier-Villars, Kent, 1985. 